\title{
Pearl and pitfalls in brain functional analysis by event-related potentials: a narrative review by the Italian Psychophysiology and Cognitive Neuroscience Society on methodological limits and clinical reliability-part II
}

\author{
Marina de Tommaso ${ }^{1} \cdot$ Viviana Betti $^{2,3} \cdot$ Tommaso Bocci $^{4} \cdot$ Nadia Bolognini $^{5,6} \cdot$ Francesco Di Russo $^{7}$. \\ Francesco Fattapposta ${ }^{8}$ - Raffaele Ferri ${ }^{9}$ - Sara Invitto ${ }^{10}$ - Giacomo Koch ${ }^{3,11}$ - Carlo Miniussi $^{12,13}$. \\ Francesco Piccione ${ }^{14}$. Aldo Ragazzoni ${ }^{15}$. Ferdinando Sartucci ${ }^{16,17}$. Simone Rossi ${ }^{18}$ - Massimiliano Valeriani ${ }^{19,20}$ (iD
}

Received: 2 November 2019 / Accepted: 21 June 2020

(C) Fondazione Società Italiana di Neurologia 2020

\begin{abstract}
This review focuses on new and/or less standardized event-related potentials methods, in order to improve their knowledge for future clinical applications. The olfactory event-related potentials (OERPs) assess the olfactory functions in time domain, with potential utility in anosmia and degenerative diseases. The transcranial magnetic stimulation-electroencephalography (TMSEEG) could support the investigation of the intracerebral connections with very high temporal discrimination. Its application in the diagnosis of disorders of consciousness has achieved recent confirmation. Magnetoencephalography (MEG) and eventrelated fields (ERF) could improve spatial accuracy of scalp signals, with potential large application in pre-surgical study of epileptic patients. Although these techniques have methodological limits, such as high inter- and intraindividual variability and high costs, their diffusion among researchers and clinicians is hopeful, pending their standardization.
\end{abstract}

Keywords Event-related potentials $\cdot$ Olfactory-evoked potentials $\cdot$ TMS-EEG $\cdot$ Event-related fields $\cdot$ Magnetoencephalography Limits $\cdot$ Reliability $\cdot$ Clinical application

Massimiliano Valeriani

massimiliano.valeriani@opbg.net

$1 \quad$ Applied Neurophysiology and Pain Unit-AnpLab-University of Bari Aldo Moro, Bari, Italy

2 Department of Psychology, Sapienza University of Rome, Rome, Italy

3 Fondazione Santa Lucia, Istituto Di Ricovero e Cura a Carattere Scientifico, Rome, Italy

4 Dipartimento di Scienze della Salute, University of Milano, Milan, Italy

5 Department of Psychology \& NeuroMi, University of Milano Bicocca, Milan, Italy

6 Laboratory of Neuropsychology, IRCCS Istituto Auxologico, Milan, Italy

7 Dept. of Movement, Human and Health Sciences, University of Rome "Foro Italico", Rome, Italy

8 Department of Human Neuroscience, Sapienza University of Rome, Rome, Italy

9 Oasi Research Institute -IRCCS, Troina, Italy
10 INSPIRE - Laboratory of Cognitive and Psychophysiological Olfactory Processes, University of Salento, Lecce, Italy

11 Neuroscience Department, Policlinico Tor Vergata, Rome, Italy

12 Center for Mind/Brain Sciences - CIMeC, University of Trento, Rovereto, Italy

13 Cognitive Neuroscience Section, IRCCS Istituto Centro San Giovanni di Dio Fatebenefratelli, Brescia, Italy

14 Brain Imaging and Neural Dynamics Research Group, IRCCS San Camillo Hospital, Venice, Italy

15 Unit of Neurology and Clinical Neurophysiology, Fondazione PAS, Scandicci, Florence, Italy

16 Section of Neurophysiopathology, Department of Clinical and Experimental Medicine, University of Pisa, Pisa, Italy

17 CNR Institute of Neuroscience, Pisa, Italy

18 Department of Medicine, Surgery and Neuroscience Siena Brain Investigation and Neuromodulation LAb (SI-BIN Lab), University of Siena, Siena, Italy

19 Neurology Unit, Bambino Gesù Hospital, Rome, Italy

20 Center for Sensory-Motor Interaction, Aalborg University, Aalborg, Denmark 


\section{Introduction}

In the last years, innovative methods of functional analysis by means of bioelectrical and biomagnetic signals have been applied to the study of normal and diseased brain. These methods refer to sensory systems of difficult approach due to the anatomical and physiological characteristics, as the olfactory pathways, whose assessment may become pivotal in the anosmic or dysosmic syndromes, in neurodegenerative diseases and chronic disorders of consciousness.

The TMS-EEG and TMS-TEP (TMS-evoked potentials) are a reliable method to investigate cortical reactivity and functional cortico-cortical connectivity. This method can be applied to different cortical targets, as the motor and dorsolateral prefrontal cortex. It has been increasingly used in many pathological conditions, ranging from psychiatric disorders to several neurologic diseases as dementia, stroke, epilepsy, and disorders of consciousness.

The last chapter is dedicated to the event-related magnetic fields (ERFs) and the potential utility of magnetic sensors in localizing main sources of cortical responses.

All these methods are still not well validated in normal and pathological populations, but the present review aims to increase their knowledge and the general interest around these innovative tools for brain functional analysis.

\section{Chemosensory event-related potentials: olfactory event-related potentials}

General description The chemosensory event-related potentials (CSERPs) are a macro-grouping including the olfactory event-related potential (OERP), the gustatory responses (GEPs), and the olfactory-trigeminal stimulation (called generally CSERP). They are a useful tool to investigate the olfactory and gustatory responses to the chemical stimulant (odorant) $[1,2]$. The distinction of the terms indicates the value of the chemosensory stimulus used. Since the 70th years, the detection of ERP components related to chemosensory stimuli was considered particularly difficult due to the inadequacy of the equipment suitable for olfactory and gustatory stimulation. However, in the early 1970, Kobal presented a first method for the controlled presentation of odorous stimuli, thus leading to the possibility of analyzing olfactory [3] and gustatory [4] CSERPs.

Several methodologies have been then applied and refined, employing a type of stimulation time-related to the ongoing EEG [5] for the extraction of event-related responses (Table 1).

Methods of recording and analysis The OERPs are a peculiar method of investigation to evaluate the olfactory functions [6]. The presence of an OERP clearly indicates the integrity of olfactory functions [7]. The method of administration of the olfactory stimuli, during an OERP or CSERP protocol, can be either central or lateralized, depending on the functions to be studied. The recording methods are different, even if the reference methodology has to be adapted to the EEG triggering in a reasonable time [8]. The minimum number of electrodes for OERP registration is three (i.e., $\mathrm{Fz}, \mathrm{Cz}, \mathrm{Pz}$ ) referenced monopolarly to the linked mastoid and grounded to the forehead $[9,10]$. The passband filters are $0.01-30 \mathrm{~Hz}, 6 \mathrm{~dB} /$ octave [9]. The OERPs or the CSERPs can be obtained averaging 10-30 artifact-free EEG epochs [10].

The same methodology is required for the gustatoryevoked potential.

Normative data The OERP component is usually a complex, slow-latent sensory component [11]. The main components detected could be identified as N1 and late positive component (LPC) [12].

The $\mathrm{N} 1$ component is probably related to the processing of the exogenous stimulus in relation to the endogenous state of the subject [13]. In fact, this component seems to vary in amplitude in relation to the concentration of odorants which co-activate the trigeminal system, while the latency is changed in dependence with different concentrations of pure odorants (the greater the concentration, the lower the latency). Furthermore, unlike the other sensory modalities, the effects of selective attention on odors lead to a reduction in latency instead of an increase in the N1 amplitude. The neuronal origins of the olfactory component N1 seem to be linked to the para-insular cortex [14].

The LPC component is probably connected to the information processing. It can be comparable to the ERP P3. Furthermore, LPC is related with the perceptual and cognitive aspects of the olfactory task [12] (Fig. 1).

The OERP normative data are highly variable, as OERPs, more than other sensory ERPs, are susceptible to variations in amplitude and latency depending on the methodology and the experimental paradigm used [15] and the qualitative and quantitative aspects of the chemical stimulus administered [7]. Murphy et al. (2000) identified normal age- and genderrelated data using a trigeminal chemical stimulus (i.e., amyl acetate). In general, females seem to have greater OERP amplitudes than males, but this aspect can be traced back to a general and meta-sensory aspect of ERPs $[9,16,17]$. There is also an age-related linear effect on OERP amplitudes [9].

Main contribution in cognitive neurosciences and neurological diseases OERPs in recent years have been extensively investigated to conduct important scientific research. Probing the mechanisms, which are at the base of the complex system of processing chemical data from outside, was the goal that the researchers were recently addressed at. From the psychophysiological point of view, OERPs represent an active 
process, able to detect, discriminate, and identify specific olfactory molecules. Moreover, from the neural point of view, it was possible to notice that the spatial localization of an active olfactory stimulus over time, therefore also due to the location of a visual and acoustic stimulus, identifies this area as a center of integration and sensory transformation of signals in space coordinates. Further studies have also identified the central, parietal, and occipital centers as areas for olfactory identification and also as selectively recruited for visual image processes [18]. Human OERPs are applied not only in basic research but also clinically and for forensic purposes [19, 20].

Clinical applications Variations of OERP may indicate alterations of the olfactory system, which may manifest as anosmic syndromes (with different etiologies) or dysosmias, which may be related to neurodegenerative complications, psychological, psychophysiological, or physiological events [21]. Furthermore, the impairment of OERP in subjects of geriatric age with neurodegenerative diseases (i.e., Alzheimer's disease) is clear. This impairment, in addition to being an index of predictive comorbidity in neurodegenerative diseases such as Alzheimer [22-25] and Parkinson's [7, 26], may also occurr in cases of primary aphasia [27]. OERP variations in elderly subjects show a deficit in detecting odors and recognizing them. Furthermore, OERPs can have relevant applications both in the field of assessment of head injuries [7, 28, 29] and in the evaluation of vegetative states [30], since the olfactory stimulus does not provide a necessarily conscious attentional channel/focus. Moreover, recent research is evaluating the effects of olfactory reactions as indicators of depression [31], in post-traumatic stress disorder [32,33] and schizophrenia [34-36].

Limits In the analysis of the olfactory cortical responses, some specific methodological biases must be taken into account. A common mode of administration of the olfactory stimulus consists in a mono-lateralized manner (that is in one nostril), but this modality can produce cortical lateralization bias both due to tactile stimulation and due to the odorous stimulation itself [37]. Furthermore, some responses to odorous stimuli can stimulate the trigeminal system, rather than the olfactory system. For example, with stimuli such as ammonia, acids, or carbon dioxide, the trigeminal system and the subcortical components are associated with nociception [38, 39]. As with other sensory modalities, the sense of smell is also susceptible to changes depending on age [40,41], stimulation [42], and tasks [23]; these variations can be viewed through the EEG. The first pilot study on EEG and olfaction was carried out by Moncrieff in 1962 [43]: this study involved the administration of an odorant on absorbent paper while the subject had his eyes closed (he was in Alpha rhythm) and evaluated the EEG cortical modification (Alpha rhythm change).
At a methodological level, presenting a direct odor on absorbent paper can have obvious disadvantages as regards the correct registration, as it can generate experimental biases, for example the presence of other types of odors in the room where the experiment examination is performed [27]. Instead, it is extremely important to be able to quantitatively check the values for odorous administration. Inter-stimulation intervals (washing) must be included in order to obtain the annulment of the tested stimulus in the environment and to avoid the habituation of the olfactory receptors (which saturate very quickly) $[44,45]$.

Once the odor is administered, the subject automatically searches, even if the task does not require it, to label the various smells perceived (naming action) [46], thus producing different ERP components depending on the encoding of the stimulus or the level of discrimination and recognition [47]. Even using new or scarcely known smells could cause the same inconvenience as the subject tries to identify them. The best solution, according to Loring [23], would be the use of a second cognitive task (masking task) such as, for example, a serial subtraction.

In the "classic" method, it is necessary to use a high control between the various experimental conditions regarding the presentation of odors or non-smells (e.g., neutral and/or baseline odor) [48]. For example, with the vials, the experimenter could create bias depending on the distance of the test tube, thus on the intensity of the stimulation, or on the motor actions of the experimenter, observed by the subject [23].

Additional bias can be produced by the type of odors presented: pleasant smells produce slow, broad breaths, while unpleasant short-term smells cause contractions of facial muscles and, consequently, artifact activities. Furthermore, it is very difficult to extract normative values. In fact, there is often a strong interaction between trigeminal aspects and purely chemioceptive olfactory aspects, which seem to balance each other. For example, patients with recently acquired anosmia show decreased trigeminal CSERP in respect to patients with older anosmia [49], while in the case of very old anosmia, the amplitude of trigeminal CSERP was found increased [50].

Perspectives The study of CSERP can be implemented, pending the solution of criticism in controlling chemical stimulation. Today, with the development of new technologies, this goal seems to be very close $[13,51,52]$.

The development of this topic is very promising, in basic and clinical research, and neuro-marketing. In fact, smell and taste, more than other senses, are particularly linked to metabolic and neuroendocrine aspects, and the accurate investigation of these aspects can lead to the understanding of the function of the central-peripheral psychophysiological bridge. 


\section{TMS-EEG}

General description Since its introduction [53], transcranial magnetic stimulation (TMS) has been considered a tool of election to study the human brain neurophysiology. It is well established how a single TMS pulse is able to activate, in the first instance, the local cortical tissue through electric currents generated by a magnetic field around the coil, and subsequently to generate an activation spreading to remote cortical areas through anatomical and functional connections. Therefore, the TMS pulse inducing electric currents in the brain and transynaptically depolarizing the cell membranes, by means of the opening of voltage-sensitive ion channels, is able to determine an action potential visible in terms of a corticalevoked potential as a post-synaptic activation outcome [54]. These cortical phenomena can be observed and investigated by simultaneously recording the EEG signal. In the last two decades, TMS-EEG has been proposed as an ideal tool to investigate cortical reactivity and functional cortico-cortical connectivity (Table 1).

Recording methods and analysis Local and at-distance cortical responses induced by the TMS pulse are topographically specified and spatiotemporally characterized by a sequence of neural events, occurring at particular latencies and with specific polarities, starting a few milliseconds after the TMS pulse and providing useful estimations of intrinsic brain mechanisms [54-57].

In clinical populations, one of the main advantages of using TMS-EEG is the possibility to assess, at the same time, different neurophysiological aspects of the stimulated cortical area. This is possible thanks to the recording of the TMSevoked potential (TEP) structure, which is a complex waveform, time-locked to the TMS pulse, formed by several negative/positive peaks lasting up to $200 \mathrm{~ms}$.

One of the most common approaches to quantify TEPs is to measure the amplitude and latency of their peaks over predefined subsets of electrodes, the so-called Electrodes $O f$ Interest (EOIs). An alternative to assess TEP peak amplitudes and latencies is to measure the area under the curve of the rectified signal corresponding to TEP peaks. This measure has been called either global or local mean field power (GMFP/LMFP), according to whether signal from all electrodes or only from a smaller cluster of electrodes is considered $[58,59]$, or cortical-evoked activity (CEA) [60]. Conversely, cluster-based permutation statistics can be applied to study signal differences over the whole scalp and TEP duration, without an a priori definition of specific clusters and latencies of interest [61, 62]. Recently, the Perturbational Complexity Index has been also introduced, which is a measure able to characterize the complexity of TMS-evoked brain responses [63].

At the same time, TMS-induced effects may be evaluated in terms of cortical oscillations, providing an estimate of the underlying brain rhythms in the target region [64]. Investigations of cortical oscillations focus on the study of TMS-induced effects in the frequency domain, as TMS is thought to interact with local and distant cortical oscillations. In this perspective, TMS-EEG offers the chance to measure activity in different frequency bands directly evoked by the TMS pulse, which is represented as a brief period of alignment of the oscillatory activity following the TMS pulse [65]. This approach that probably reflects the physiological organization of thalamo-cortical brain columns is useful to identify the specific frequency characterizing the functional status of the stimulated brain area [57].

Normative data TEP waveforms vary when evoked over different areas, such as the primary motor cortex (M1), the dorsolateral prefrontal cortex (DLPFC), or the posterior parietal cortex (PPC), thus providing specific information on local neural activity [66]. For instance, TEPs in M1 (Fig. 2) are characterized by the peaks N15, P30, N45, P55, N100, $\mathrm{P} 180$, and $\mathrm{N} 280[67,68]$. It has been suggested that when TMS is applied over the left M1, the initial N15 peak occurs in electrodes over the stimulated area, and the peak distribution then spreads over central parietal sites (P30) and contralateral regions (N45). A bilateral distribution over temporocentral sites characterizes later TEP components, peaking at $100 \mathrm{~ms}$ and $180 \mathrm{~ms}$, respectively. However, these later peaks are influenced by the acoustic artifact of the discharging coil $[69,70]$, which should be covered by a white noise, reproducing shuffled time frequency components of the coil discharge sound, during the TMS/EEG recording [71]. Paired-pulse and pharmacological studies have also suggested that early peaks (N15-P30) are likely to reflect cortical excitatory activity, while later peaks (N45-N100) are primarily linked to GABAergic inhibition [72].

Apart from M1, TEPs have also been characterized in the DLPFC, showing P25, N40, P60, N100, and P185 peaks, that may be smaller in amplitude as compared to those in M1 [68]. Several other areas have been tested, including the premotor cortex [73-76], supplementary motor area [75], parietal cortex [76-78], and the occipital cortex [57, 79]. However, there is still no consensus on the standardization required to define the characteristics of these TEPs. Data from normal controls, standardized for age and gender, are still unavailable, as TEPs were obtained in small cohorts of healthy subjects and neurological patients.

Main contribution in cognitive neurosciences and neurological diseases The combined TMS-EEG approach can be used to evaluate the spread of cortical-evoked activity across specific brain networks [80,81]; this is relevant in order to define specific patterns of altered connectivity in different clinical populations. When paired-pulse TMS is applied over nonmotor areas, such as the DLPFC, it is also possible to assess 
the integrity of local intracortical circuits, likely involving GABAergic activity [82]. Finally, it is possible to investigate plasticity within specific cortical circuits, for instance by repeatedly applying magnetic stimuli over two interconnected regions and measuring the after-effects of this paired associative stimulation [83, 84].

Clinical applications Clearly, from the clinical point of view, such abundance of information can help to characterize the functional abnormalities of certain brain areas in a given pathology. Therefore, TMS-EEG can become useful for differential diagnosis, to predict the outcome and the progression of a particular disease and, eventually, to verify the response to drug treatment, or to neuromodulation protocols.

As a consequence of this tremendous - although yet not standardized - potential, TMS-EEG has been increasingly used in a broad range of psychiatric and neurological disorders, including major depression, schizophrenia, addiction, autism, dementia, stroke, epilepsy, and disorders of consciousness [62]; in the latter case, TMS-EEG seems even the most sensitive neurophysiological approache to differentiate patients with prolonged disorders of consciousness, at the bedside [77, 85].

Other emerging clinical applications of TMS-EEG include mood disorders: in this case, TMS-EEG has been shown useful to predict remission following therapy [86] and to monitor the effects of non-pharmacological therapies such as electroconvulsive therapy [87] and repetitive TMS [88].

In patients with stroke, TMS-EEG has provided novel neurophysiological indexes of cortical reorganization after stroke [89]. In Alzheimer's disease, TMS-EEG has been used to map changes in cortical activity induced by therapeutic neuromodulation [70]; moreover, TMS-EEG-evoked component P30 may predict cognitive scores: higher P30 amplitudes appear to be associated with greater cognitive and memory impairments [90].

Limits There are several technical problems that still have not been fully overcome. Artifact correction has to be further implemented [91, 92]. Moreover, a major potential confound is due to the fact that a TMS pulse produces a loud clicking noise $(100-120 \mathrm{~dB})$ that generates an auditory-evoked potential, potentially contaminating the underlying TMS-evoked activity [61]. In addition, the TMS pulse can induce unwanted somatosensory-evoked potentials that could contribute to the signal [93]. Post-processing of the EEG signal is also an important step in the data analysis that needs to be standardized across labs [94, 95].

Perspectives Even if this is still an emerging methodological field, the TMS-EEG approach is also valuable as control system for neuromodulatory approaches [96] by means of an open- or closed-loop method. In this paradigm, the TMS input to the brain is delivered at a predefined set-point based on the EEG data. Thus, we can manipulate the inputs at a given moment to obtain the desired/predicted effect on the output of the system. Moreover, by monitoring the parameter to provide feedback and adjusting the control signal, information about the precise state of the system can be derived. Therefore, by means of specific TMS frequencies or intensities, it is possible to facilitate, suppress, or maintain that brain state within defined ranges. This evidence highlights the intrinsic clinical potential of TMS-EEG for diagnosis and rehabilitation.

\section{Event-related magnetic fields}

Definition Brain functioning is associated with primary electric currents generated by the post-synaptic potentials in the cortical pyramidal neurons. These neuronal currents give rise to both voltage differences measurable on the scalp by means of electroencephalography and magnetic fields detectable outside the head by using magnetoencephalography [97] (Fig. 3). For this reason, both EEG and MEG are considered real-time non-invasive methods to characterize directly event-related brain dynamics. The fact that EEG and MEG share similar neurophysiological mechanisms has fueled the misconception that MEG is simply redundant with EEG; MEG offers several advantages [98]. In this chapter, we will focus on basic aspects featuring MEG as compared to EEG, and particularly we will describe how its application can help in elucidating the brain mechanisms underlying normal physiological and pathological brain functions (Table 1).

Recording methods and analysis In order to extract relevant signals, MEG relies on a sophisticated sensing technology, which is sensible to minuscule changes in the magnetic fields produced by the small changes in electrical activity within the brain. Indeed, extracranial magnetic induction is on scale of femtoteslas $\left(1 \mathrm{fT}=10^{-15} \mathrm{~T}\right)$, about 10 to 100 million times smaller than the Earth's static magnetic field [99]. This imposes the need of superconducting quantum interference devices (SQUIDs), which are sensors arranged in arrays around the head that, by becoming superconductors when cooled down to very low temperatures (close to the $0^{\circ} \mathrm{Kelvin}$ ), warrant very high data quality. Given the necessity of very low temperatures, SQUIDs are not placed at direct contact with the head (as for the EEG setup) but they are immersed within a large, vacuum-insulated dewar, which contains liquid helium. To detect the neuronal activity propagating from the brain to the scalp level, SQUIDs are coupled to flux transformers, which are other superconducting components capable of concentrating the magnetic signal [100], and composed by a signal coil, a compensation coil (optional), and a pickup coil. Signal coils are placed close to the SQUIDs and connected 


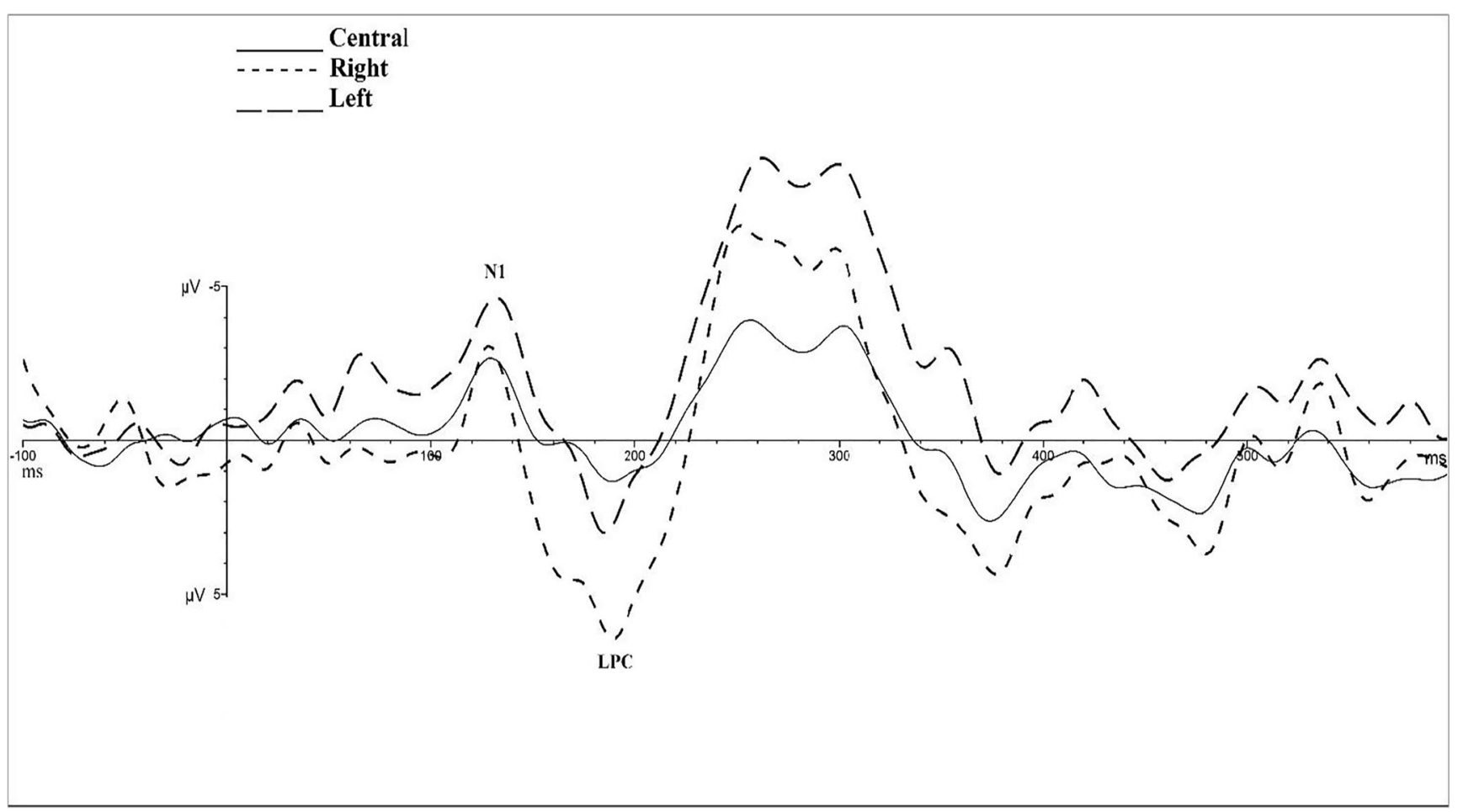

Fig. 1 Averaged OERP waveform comparison between central, right, and left EEG regions of interest (ROI) during a perceptual task with PEA stimulations in a healthy subject

to the compensation coils. These latter are connected to the pickup coils, which are placed inside the MEG helmet, where the participant's head is positioned to record the brain activity. The simplest type of MEG sensor is the magnetometer, which consists of a pickup coil without a compensation coil. Many MEG systems are based on gradiometers, consisting of two coupled coils. The spatial arrangement of these two coils has important consequences on how the signal is detected at the sensor level, and then is visualized. In axial gradiometers, the two coils are arranged one on top of the other (with the pickup coil closer to the head), while in planar gradiometers, the two coils are placed one beside the other. Thus, an evoked activity to an auditory stimulus could be seen as both positive and negative peaks in nearby sensors in axial gradiometers (with the generating electrical current between these positive and negative peaks) or as positive peaks in planar gradiometers (with the generating electrical current in the middle of these positive peaks) [100].

In MEG literature, responses to sensory stimuli or active tasks, occurring at the same time and phase-locked, are referred to as event-related fields (ERFs). ERFs represent the magnetic correspondence of event-related potentials (ERPs). Therefore, it is not surprising that the methodological approaches to process and analyze MEG data largely overlap with those employed in EEG research. In the time domain, changes in brain activity can be appreciated through analysis of amplitude and latency; the final result is that most ERP components have their magnetic counterpart (e.g., N400m or MMNm). In the frequency domain, the same mathematical approaches to spectrally decompose the EEG signal are also available for MEG data. At the source level, since MEG has higher spatial resolution compared to EEG, greater effort has

Table 1 Advantages, limitations, and clinical applications of the psychophysiological techniques - part II

\begin{tabular}{|c|c|c|c|}
\hline Technique & Advantages & Limitations & Clinical applications \\
\hline OERPs & $\begin{array}{l}\text { Time domain assessment of the } \\
\text { olfactory pathway }\end{array}$ & $\begin{array}{l}\text { Large inter- and intra-subject variability, } \\
\text { weak specificity of the stimulus }\end{array}$ & Anosmia, dysosmia, dementia \\
\hline TMS-EEG & $\begin{array}{l}\text { Investigation of the intracerebral } \\
\text { connections with very high } \\
\text { temporal discrimination }\end{array}$ & $\begin{array}{l}\text { Technical issues (contamination by } \\
\text { acoustic and somatosensory responses) }\end{array}$ & Disorders of consciousness, stroke, dementia \\
\hline MEG & Powerful spatial discrimination & $\begin{array}{l}\text { Cost of the equipment, weak sensitivity } \\
\text { to radial sources }\end{array}$ & Epilepsy, pre-surgical brain mapping \\
\hline
\end{tabular}


been made to develop mathematical solutions to localize brain sources. This explains why, in many EEG studies, MEG is considered a ground truth and used for comparison to evaluate the quality of the localization results.

Advantages and limits MEG, like EEG, has the intrinsic limitation that the neural signals are only recorded from the scalp. However, since MEG measures extracranial magnetic fields about $20 \mathrm{~mm}$ above the scalp, and not voltage differences, it is immune to both the reference problem and the volume conduction issue. Thus, the estimation of the inverse solution, i.e., the most likely neural sources generating the magnetic field, is more straightforward as compared to EEG. The distortion of the electric current produced by the inhomogeneities of the media (Fig. 3a) can be appreciated, at the sensor level, as widespread electric potential distribution on the scalp much more than for the magnetic field, despite similarities in the spectral content of the cortical activity between EEG and MEG (Fig. 3b). At the source level, MEG is blind to radially oriented fields, since they do not generate a magnetic field outside the head. By contrast, MEG is highly sensitive to tangentially oriented dipolar fields, which are associated with the neural activity occurring in the walls of the cortical fissures. This is an advantage, as about two-thirds of the cerebral cortex is located within fissures, including all the primary sensory cortices, which are difficult to reach even with intracranial recordings [99].

The strong focus on the source level analysis in the MEG literature can be explained also by some major drawbacks intrinsic to the sensor level analysis. The first one is related to the MEG helmet. While in EEG sensors are placed according to head size, and move with the subject's head, in MEG, sensors are fixed in a rigid position. This means that the head shape, size, and position during the recording actually affect what a given sensor is measuring [98]. A second disadvantage is related to the polarity of the recorded ERF components. Whereas in EEG there is a major agreement on the use of specific reference electrodes, which determines consistency in the polarity and distribution of observed ERP components, at least within each subfield of literature, in MEG, the typology of sensors influences the observed polarity of the deflections. To further complicate this issue, in some common MEG sensors (e.g., with axial gradiometers), a single electrical current is associated with dipolar distributions of the recorded magnetic fields (so with neighboring positive and negative deflections). This dipolar distribution challenges the typical scheme of classification of ERP components in terms of polarity and distribution on the scalp. Also for these reasons, most of the more recent MEG studies using ERF rely solely on the source reconstruction of the signal, and focus on brain sources $[101,102]$.

ERF studies on MEG Most of the MEG paradigms used in basic research for cognitive neuroscience are strictly related to the EEG literature. This is not surprising, given the similarity between the signals from the two methodologies. A quick inspection of the first studies on MEG shows that they were basically adaptations of EEG studies. By exploiting the main advantage of MEG (i.e., the higher spatial accuracy), these first studies mostly tried to localize the neural sources of the MEG equivalent of known ERP components (usually labeled to underline their magnetic origin: e.g., $\mathrm{N} 400 \mathrm{~m}$ or $\mathrm{MMNm}$ ).

One of the most studied ERF components in MEG literature is the magnetic equivalent of the mismatch negativity, the MMNm [103-105]. EEG MMN is observed typically in auditory tasks in which a series of frequent standard stimuli are a

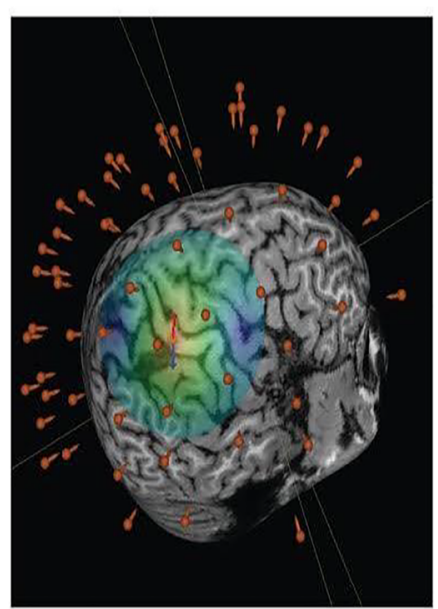

b

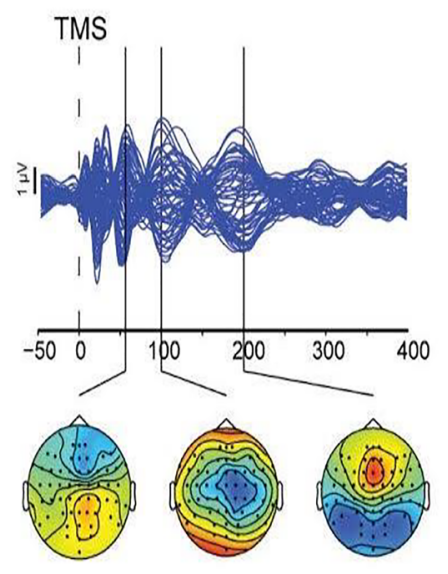

C

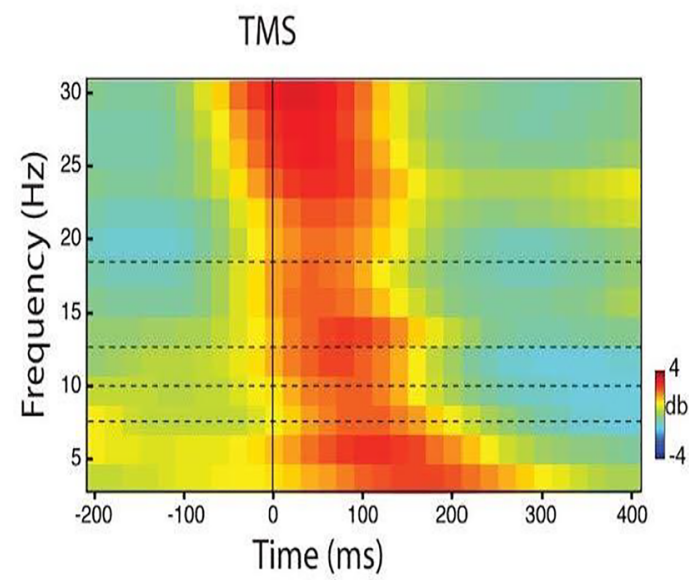

Fig. 2 a An example of a neuronavigated TMS-EEG session showing sensor location (orange dots), TMS hotspot (red/blue arrow), and induced electrical field. b Butterfly plot depicting TEPs evoked in 60 EEG channels (upper row) and scalp topographies of the main evoked components (lower row). c Time frequency plot of a TMS-EEG response. (Adapted from Pisoni et al. 2018 [62]) 


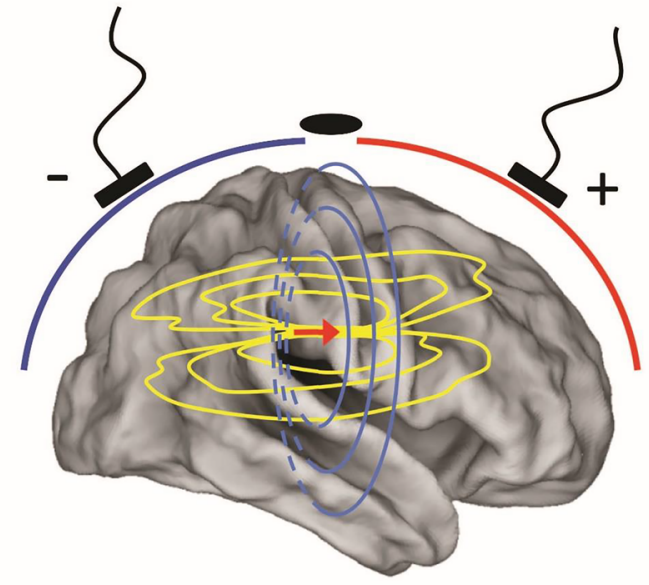

(a)

Fig. 3 Origin of EEG and MEG signals. a Schematic representation of electrical current (red arrow) within the head, generated from a patch of cortex. b Two comparable brain components, computed with independent component analysis (ICA), from EEG and MEG data,

interspersed with (less frequent) deviant stimuli. MMN is computed by subtracting the ERP associated with deviant stimuli to the ones associated with the standard stimuli. The MMNm component is observed in analogous tasks, with MEG recordings. MMNm typically peaks around $130 \mathrm{~ms}$ after the stimulus, but its timing could depend on the type of task [104]. The localized MMNm shows bilateral temporal activation, especially in the supratemporal auditory cortices [106], with higher amplitude in the right hemisphere [107].

Another ERF component that has been largely investigated in MEG studies is the N400m.

EEG N400 is typically observed in language tasks, and it is a component associated with incongruence. In a typical language task to elicit N400, a sequence of letter is presented and the ERP is measured with a target word that could be congruent or not with the preceding words. Incongruent targets show higher negative amplitude as compared to the congruent ones, with a wave peaking at about $400 \mathrm{~ms}$. In a study with EEG and MEG co-registration, Simos, Basile, and Papanicolaou (1997) found that in the same time window of the N400, MEG showed deflections mostly localized in the left temporal cortex.

ERF components have been also studied to investigate more automatic responses, as the magnetic equivalent of the N20. The EEG N20 peak is observed after median nerve stimulation with electrical stimulation delivered at the wrist. The MEG equivalent, the $\mathrm{N} 20 \mathrm{~m}$, is observed in the same time window. MEG studies on N20m have been used to estimate, as accurately as possible, the loci of the neural generators of this component [108].

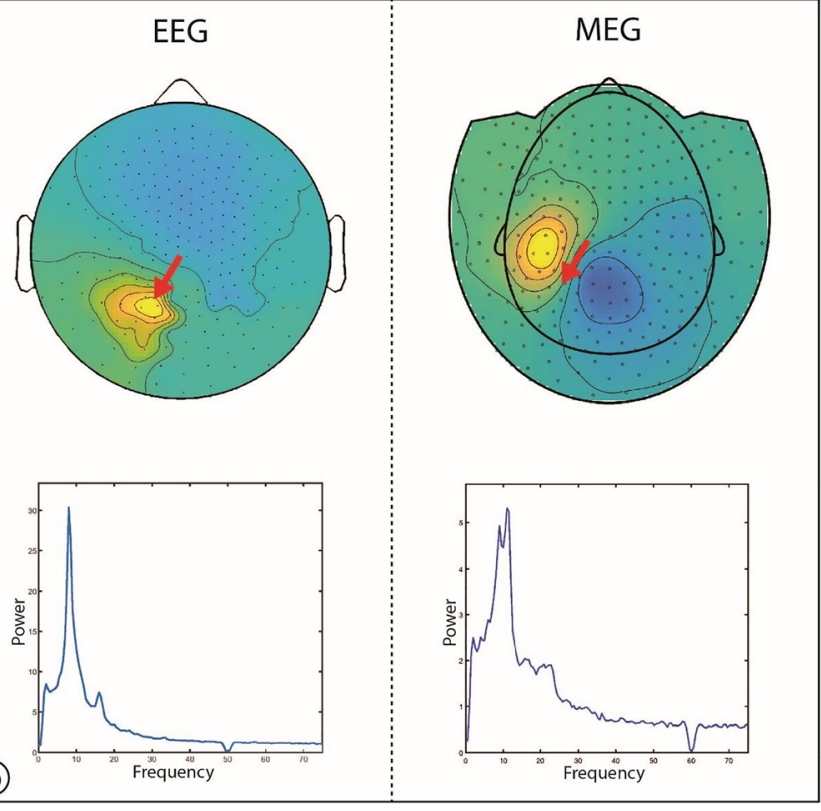

respectively. Both fields are dipolar (red arrow) but the topographies (top) show tighter isocontour lines for MEG as compared to EEG, due to the influence of the inhomogeneities that smear out the electric potential much more than in the magnetic field

The investigation of ERFs allowed not only to characterize the neural generators, but also to better understand the complexity of some EEG components. This is for example the case for the studies of the magnetic equivalent of the P300. EEG. P300 is a broad positivity observed in all modalities after rare and task-salient stimuli. Its latency typically is around $300 \mathrm{~ms}$, but it varies depending on task and subject [109]. Within P300, we can distinguish two components, the $\mathrm{P} 3 \mathrm{a}$, associated with stimulus-driven novelty, and the P3b, mostly associated with attentional and memory processing [110]. MEG equivalent of P300, P300m, clearly unveils the complexity underlying the apparent unitarity of this component. First of all, in MEG studies, P300m tends to be less evident already at sensor level [111], as compared to the EEG, and it is associated with at least two separated peaks in time. The neural generators associated with the $\mathrm{P} 300 \mathrm{~m}$ are diffuse, and include medial temporal lobes, but also parietal and prefrontal regions [112]. Interestingly, despite the higher spatial resolution, most of the studies on the P300 source localization do not rely on MEG data, but are based on the EEG P300.

Clinical application of MEG The specific advantages of MEG (i.e., less distortion from volume conduction, higher number of sensors, short preparation time) make it the perfect technique for the accurate spatial localization of electromagnetic activity of the brain also for clinical purposes. According to the existing guidelines for the use of MEG for clinical applications, there are two main ways of using MEG: the study of 
spontaneous activity or the study of evoked activity [113-115]. Concerning the spontaneous activity, MEG is mainly used for the localization of epileptic spikes. In the case of patients with drug-resistant epilepsy, in which a neurosurgical treatment is the only viable option, it is of major importance to know where the seizures are generated (if there is a focal epileptogenic area) and the extension of this area [116, 117]. Knowing these details, the neurosurgeon can guide the surgical operation and improve the prognosis for the patient. The current evidence suggests that MEG can be very accurate (as compared to intracortical recordings that are the gold standard) and can outperform EEG in terms of accuracy [118].

Concerning the evoked activity, MEG is mainly used for mapping eloquent areas in patients with brain tumor before undergoing neurosurgery. Usually, mapping concerns the localization of language areas (either simple lateralization or more accurate mapping), or, less frequently, the localization of areas involved in motor functions. Concerning language mapping, MEG has some important advantages as compared with functional magnetic resonance imaging (fMRI), which is the most common technique used for pre-surgical mapping. Despite the widespread use of fMRI for mapping, the modifications in the vascular coupling that happens in brain tumors (especially in the vicinity of tumor) can affect the reliability of BOLD signal, making the technique potentially inaccurate [119]. Being a more direct measure of brain activity, and given its good spatial accuracy, MEG is a valid alternative for presurgical mapping. There are several tasks that can be used in MEG for language mapping [120, 121], but the most common ones include picture naming or verb generation $[122,123]$. In the current clinical practice of MEG, ERFs still play a crucial role. The American Magnetoencephalographic Association focused its guidelines on ERF responses, either evoked by external stimuli or by endogenous epileptic spikes [113, 114]. The relevance of ERF as a robust technique is also stressed by the more recent IFCN endorsed guideline for clinical MEG [99]. However, as described in the next paragraph, recent trends are moving toward more advanced analysis, mostly focused on the study of frequency-specific activity and responses.

\section{Current and future developments of MEG application} According to recent developments of basic and clinical research, an increasing number of MEG studies is gradually moving beyond simple ERFs analysis, toward more sophisticated analysis strategies focusing on MEG activity and connectivity in specific frequency bands, during resting state and active tasks [124, 125]. Recently, MEG has been fruitfully used to understand how brain connectivity changes after tDCS, by taking advantage of MEG short preparation time and insensitivity to tDCS gel [126], while other studies showed abnormal patterns of connectivity in clinical populations as compared with controls [127]. Also, besides these common clinical purposes, MEG can be effectively used for rehabilitation. Recent MEG-based BCI application has been developed to assist and improve recovery in stroke patients [128]. Lastly, the future of MEG research is oriented to the identification of diagnostic markers that can yield new clinical applications, especially for monitoring the functional recovery in patients with brain injury and assessing the progression of neurodegenerative diseases.

\section{General remarks}

In the second part of this review article, we dealt with psychophysiological techniques which are less currently used in the clinical practice, as compared to the ERP components examined in the first part. This does not depend only on the lack of evidence of their utility in diseases, but also, especially for MEG, on other reasons. Indeed, the advantage that MEG investigation can provide in some clinical situations, such as the pre-surgical assessment of epilepsies, is well documented. However, the high cost of the equipment does not allow a large diffusion of this psychophysiological method. As for the OERPs and TMS-EEG recording, their use is still limited to the research field, although their possible utility also in patients can be guessed from the presently available data. The extraordinary progress which have been obtained in the comprehension of the brain mechanisms in some disorders of consciousness through TMS-EEG suggests that it could provide important diagnostic and prognostic data in this condition.

In conclusion, we are convinced that, in spite of the continuous updating of the neuroimaging techniques, ERP recording should still be part of the neurological and psychological culture, not as a memory of the past, but as a tool able to understand the brain functioning in healthy subjects and diseases.

Author contribution Marina de Tommaso: introduction, manuscript design, and editing

Massimiliano Valeriani: conclusions, manuscript design, and editing

Viviana Betti, Francesco Piccione, Giorgio Arcara, Marco Marino: manuscript editing, event-related field, and magnetoencephalogram chapter

Sara Invitto and Ferdinando Sartucci: manuscript editing, olfactoryevoked potentials chapter

Aldo Ragazzoni: manuscript editing

Francesco Fattapposta: manuscript editing

Raffaele Ferri: manuscript editing

Giacomo Koch: manuscript editing, TMS-EEG chapter

Francesco Di Russo: manuscript editing

Tommaso Bocci: manuscript editing; Carlo Miniussi: manuscript editing; Nadia Bolognini: manuscript editing; Valentina Bianco: manuscript editing; Marianna Delussi: manuscript editing; Eleonora Gentile: manuscript editing; Fabio Giovannelli: manuscript editing; Daniela Mannarelli: manuscript editing; Elena Mussini: manuscript editing; Caterina Pauletti: manuscript editing; Maria COncetta Pellicciari: manuscript editing; Alberto Pisoni: manuscript editing; Alberto Raggi: manuscript editing 


\section{Compliance with ethical standards}

Conflict of interest The authors declare that they have no conflict of interest.

\section{References}

1. Kobal G, Hummel T (1994) Olfactory (chemosensory) eventrelated potentials. Toxicol Ind Health 10:587-596

2. Landis BN, Negoias S, Friedrich H (2016) Chemosensorisch evozierte Potenziale chemosensory event related potentials. Epileptologie 33:189-196

3. Kobal G, Plattig KH (1978) Objective olfactometry: methodological annotations for recording olfactory EEG-responses from the awake human. Elektroenzephalogr Elektromyogr Verwandte Geb 9:135-145

4. Kobal G (1985) Gustatory evoked potentials in man. Electroencephalogr Clin Neurophysiol Evoked Potentials 62: 449-454. https://doi.org/10.1016/0168-5597(85)90055-3

5. Bonanni E, Borghetti D, Fabbrini M, Maestri M, Cignoni F, Sartucci F (2006) Quantitative EEG analysis in post-traumatic anosmia. Brain Res Bull 71:69-75. https://doi.org/10.1016/j. brainresbull.2006.08.004

6. Hu B, Han D, Zhang L, Li Y, Zang H, Wang T, Xian M, Zhang W, Yang L, Wang H, He F (2010) Olfactory event-related potential in patients with rhinosinusitis-induced olfactory dysfunction. Am J Rhinol Allergy 24(5):330-335. https://doi.org/10.2500/ajra. 2010.24.3517

7. Lötsch J, Hummel T (2006) The clinical significance of electrophysiological measures of olfactory function. Behav Brain Res 170:78-83. https://doi.org/10.1016/j.bbr.2006.02.013

8. Luck SJ (2005). An introduction to event-related potentials and their neural origins. MIT Press, Cambridge, Mass, London

9. Murphy C, Morgan CD, Geisler MW, Wetter S, Covington JW, Madowitz MD (2000) Olfactory event-related potentials and aging: normative data. Int J Psychophysiol 36:133-145

10. Rombaux P, Mouraux A, Bertrand B, Guerit J, Hummel T (2006) Assessment of olfactory and trigeminal function using chemosensory event-related potentials. Neurophysiol Clin 36: 53-62. https://doi.org/10.1016/j.neucli.2006.03.005

11. Wang L (2002) The correlation between physiological and psychological responses to odour stimulation in human subjects. Clin Neurophysiol 113:542-551

12. Pause BM, Sojka B, Krauel K, Ferstl R (1996) The nature of the late positive complex within the olfactory event- related potential (OERP). Psychophysiology 33:376-384. https://doi.org/10.1111/ j.1469-8986.1996.tb01062.x

13. Pause BM, Krauel K (2000) Chemosensory event-related potentials (CSERP) as a key to the psychology of odors. Int $\mathbf{J}$ Psychophysiol 36:105-122. https://doi.org/10.1016/S01678760(99)00105-1

14. Kettenmann B, Hummel C, Stefan H, Kobal G (1997) Multiple olfactory activity in the human neocortex identified by magnetic source imaging. Chem Senses 22:493-502. https://doi.org/10. 1093/chemse/22.5.493

15. Geisler MW, Murphy C (2000) Event-related brain potentials to attended and ignored olfactory and trigeminal stimuli. Int $\mathrm{J}$ Psychophysiol 37:309-315

16. Picton TW (1992) The P300 wave of the human event-related potential. J Clin Neurophysiol 9:456-479. https://doi.org/10. 1097/00004691-199210000-00002
17. Picton TW (2000) Guidelines for using human event-related potentials to study cognition: recording standards and publication criteria. Psychophysiology 37:127-152

18. Porter J, Anand T, Johnson B, Khan RM, Sobel N (2005) Brain mechanisms for extracting spatial information from smell. Neuron 47:581-592. https://doi.org/10.1016/j.neuron.2005.06.028

19. Auffermann H, Mathe F, Gerull G, Mrowinski D (1993) Olfactory evoked potentials and contingent negative variation simultaneously recorded for diagnosis of smell disorders. Ann Otol Rhinol Laryngol 102:6-10. https://doi.org/10.1177/000348949310200102

20. Sirous M, Sinning N, Schneider TR, Friese U, Lorenz J, Engel AK (2019) Chemosensory event-related potentials in response to nasal propylene glycol stimulation. Front Hum Neurosci 13:99. https:// doi.org/10.3389/fnhum.2019.00099

21. Miwa T, Furukawa M, Tsukatani T, Costanzo RM, DiNardo LJ, Reiter ER (2001) Impact of olfactory impairment on quality of life and disability. Arch Otolaryngol Head Neck Surg 127:497-503

22. Invitto S, Piraino G, Ciccarese V, Carmillo L, Caggiula M, Trianni G (2018) Potential role of OERP as early marker of mild cognitive impairment. Front Aging Neurosci 10:272. https://doi.org/10. 3389/fnagi.2018.00272

23. Lorig TS, Schwartz GE (1988) Brain and odor: I. Alteration of human EEG by odor administration. Psychobiology. https://doi. org/10.3758/BF03327318

24. Mazzatenta A, Pokorski M, Di Tano A, Cacchio M, Di Giulio C (2016) Influence of sensory stimulation on exhaled volatile organic compounds. Adv Exp Med Biol. https://doi.org/10.1007/5584 2015176

25. Morgan CD, Murphy C (2002) Olfactory event-related potentials in Alzheimer's disease. J Int Neuropsychol Soc 8:753-763

26. Iannilli E, Stephan L, Hummel T, Reichmann H, Haehner A (2017) Olfactory impairment in Parkinson's disease is a consequence of central nervous system decline. J Neurol 264:12361246. https://doi.org/10.1007/s00415-017-8521-0

27. Lorig TS (1989) Human EEG and odor response. Prog Neurobiol 33:387-398

28. Doty RL (2012) Smell and taste. In: Sinclair AJ, Morley JE, Vellas B (eds) Pathy's principles and practice of geriatric medicine, Fifth edn. Wiley, p 1061-1072

29. Malaty J, Malaty IAC (2013) Smell and taste disorders in primary care. Am Fam Physician 88:852-859

30. Nigri A, Ferraro S, Bruzzone MG, Nava S, D'Incerti L, Bertolino $\mathrm{N}$ et al (2016) Central olfactory processing in patients with disorders of consciousness. Eur J Neurol 23:605-612. https://doi.org/ 10.1111/ene. 12907

31. Croy I, Hummel T (2017) Olfaction as a marker for depression. J Neurol 264:631-638. https://doi.org/10.1007/s00415-016-8227-8

32. Dileo JF, Brewer WJ, Hopwood M, Anderson V, Creamer M (2008) Olfactory identification dysfunction, aggression and impulsivity in war veterans with post-traumatic stress disorder. Psychol Med 38:523-531. https://doi.org/10.1017/ S0033291707001456

33. Vermetten E, Bremner JD (2003) Olfaction as a traumatic reminder in posttraumatic stress disorder: case reports and review. J Clin Psychiatry 64:202-207. https://doi.org/10.4088/JCP.v64n0214

34. Moberg PJ, Agrin R, Gur RE, Gur RC, Turetsky BI, Doty RL (1999) Olfactory dysfunction in schizophrenia: a qualitative and quantitative review. Neuropsychopharmacology. 21:325-340. https://doi.org/10.1016/S0893-133X(99)00019-6

35. Stevenson RJ (2013) Olfactory perception, cognition, and dysfunction in humans. Wiley Interdiscip Rev Cogn Sci 4. https:// doi.org/10.1002/wcs. 1224

36. Turetsky BI, Hahn CG, Borgmann-Winter K, Moberg PJ (2009) Scents and nonsense: olfactory dysfunction in schizophrenia. Schizophr Bull 35:1117-1131. https://doi.org/10.1093/schbul/ $\operatorname{sbp} 111$ 
37. Olofsson JK, Broman DA, Gilbert PE, Dean P, Nordin S, Murphy C (2006) Laterality of the olfactory event-related potential response:699-704. https://doi.org/10.1093/chemse/bj1011

38. Frasnelli J, Schuster B, Hummel T (2007) Interactions between olfaction and the trigeminal system: what can be learned from olfactory loss. Cereb Cortex 17:2268-2275

39. Hummel T, Livermore A (2002) Intranasal chemosensory function of the trigeminal nerve and aspects of its relation to olfaction. Int Arch Occup Environ Health 75:305-313

40. Attems J, Walker L, Jellinger KA (2015) Olfaction and aging: a mini-review. Gerontology 61:485-490. https://doi.org/10.1159/ 000381619

41. Schriever VA, Góis-Eanes M, Schuster B, Huart C, Hummel T (2014) Olfactory event-related potentials in infants. J Pediatr 165: 372-375.e2

42. Brauchli P, Rüegg PB, Etzweiler F, Zeier H (1995) Electrocortical and autonomic alteration by administration of a pleasant and an unpleasant odor. Chem Senses 20:505-515

43. Martin GN (2013) The neuropsychology of smell and taste. https://doi.org/10.4324/9780203070147

44. Chaudhury D, Manella L, Arellanos A, Escanilla O, Cleland TA, Linster C (2010) Olfactory bulb habituation to odor stimuli. Behav Neurosci 124:490-499

45. Poellinger A, Thomas R, Lio P, Lee A, Makris N, Rosen BR, Kwong KK (2001) Activation and habituation in olfaction-an fMRI study. Neuroimage 13:547-560. https://doi.org/10.1006/ nimg.2000.0713

46. Frank RA, Rybalsky K, Brearton M, Mannea E (2011) Odor recognition memory as a function of odor-naming performance. Chem Senses 36:29-41

47. Invitto S, Piraino G, Mignozzi A, Capone S, Montagna G, Siciliano PA et al (2017) Smell and meaning: an OERP study. Smart Innov Syst Technol:289-300. https://doi.org/10.1007/9783-319-56904-8_28

48. Invitto S, Mazzatenta A (2019) Olfactory event-related potentials and exhaled organic volatile compounds: the slow link between olfactory perception and breath metabolic response a pilot study on phenylethyl alcohol and vaseline oil. Brain Sci 9:84. https://doi. org/10.3390/brainsci9040084

49. Kobal G, Hummel T (1998) Olfactory and intranasal trigeminal event-related potentials in anosmic patients. Laryngoscope 108: 1033-1035

50. Hummel T, Kaehling C, Grosse F (2016) Automated assessment of intranasal trigeminal function. Rhinology:54. https://doi.org/10. 4193/Rhin15.002

51. Boesveldt S, Haehner A, Berendse HW, Hummel T (2007) Signal-to-noise ratio of chemosensory event-related potentials. Clin Neurophysiol 118:690-695. https://doi.org/10.1016/j. clinph.2006.11.004

52. Invitto $\mathrm{S}$, Calcagnì $\mathrm{A}$, Piraino $\mathrm{G}$, Ciccarese $\mathrm{V}$, Balconi $\mathrm{M}$, De Tommaso $\mathrm{M}$ et al (2019) Obstructive sleep apnea syndrome and olfactory perception: an OERP study. Respir Physiol Neurobiol 259:37-44. https://doi.org/10.1016/j.resp.2018.07.002

53. Barker AT, Jalinous R, Freeston IL (1985) Non-invasive magnetic stimulation of human motor cortex. Lancet 1(8437):1106-1107

54. Ilmoniemi RJ, Kičić D (2010) Methodology for combined TMS and EEG. Brain Topogr 22(4):233-248

55. Amassian VE, Cracco RQ (1987) Human cerebral cortical responses to contralateral transcranial stimulation. Neurosurgery 20(1):148-155

56. Cracco RQ, Amassian VE, Maccabee PJ, Cracco JB (1989) Comparison of human transcallosal responses evoked by magnetic coil and electrical stimulation. Electroencephalogr Clin Neurophysiol 74:417-424

57. Ilmoniemi RJ, Virtanen J, Ruohonen J, Karhu J, Aronen HJ, Näätänen R (1997) Neuronal responses to magnetic stimulation reveal cortical reactivity and connectivity. Neuroreport 8:35373540

58. Pellicciari MC, Brignani D, Miniussi C (2013) Excitability modulation of the motor system induced by transcranial direct current stimulation: a multimodal approach. Neuroimage 83:569-580. https://doi.org/10.1016/j.neuroimage.2013.06.076

59. Romero Lauro LJ, Rosanova M, Mattavelli G, Convento S, Pisoni A, Opitz A, Bolognini N, Vallar G (2014) TDCS increases cortical excitability: direct evidence from TMS-EEG. Cortex 58:99-111. https://doi.org/10.1016/j.cortex.2014.05.003

60. Rajji TK, Sun Y, Zomorrodi-Moghaddam R, Farzan F, Blumberger DM, Mulsant BH, Fitzgerald PB, Daskalakis ZJ (2013) PAS-induced potentiation of cortical-evoked activity in the dorsolateral prefrontal cortex. Neuropsychopharmacology 38(12):2545-2552. https://doi.org/10.1038/npp.2013.161

61. Mattavelli G, Pisoni A, Romero Lauro LJ, Marino BF, Bonomi M, Rosanova M, Papagno C (2019) TMSEEG approach unveils brain mechanisms underlying conscious and unconscious face perception. Brain Stimul 12(4):1010-1019. https://doi.org/10.1016/j.brs. 2019.02.022

62. Pisoni A, Romero Lauro LJ, Vergallito A, Maddaluno O, Bolognini N (2018) Cortical dynamics underpinning the selfother distinction of touch: a TMS-EEG study. Neuroimage 178: 475-484

63. Casarotto S, Comanducci A, Rosanova M, Sarasso S, Fecchio M, Napolitani M, Pigorini A, G. Casali A, Trimarchi PD, Boly M, Gosseries O, Bodart O, Curto F, Landi C, Mariotti M, Devalle G, Laureys S, Tononi G, Massimini M (2016) Stratification of unresponsive patients by an independently validated index of brain complexity. Ann Neurol 80:718-729

64. Thut G, Miniussi C (2009) New insights into rhythmic brain activity from TMS-EEG studies. Trends Cogn Sci 13:182-189

65. Thut G, Veniero D, Romei V, Miniussi C, Schyns P, Gross J (2011) Rhythmic TMS causes local entrainment of natural oscillatory signatures. Curr Biol 21(14):1176-1185. https://doi.org/10. 1016/j.cub.2011.05.049

66. Rosanova M, Casali A, Bellina V, Resta F, Mariotti M, Massimini MJ (2009) Natural frequencies of human corticothalamic circuits. Neurosci 29(24):7679-7685. https://doi.org/10.1523/ JNEUROSCI.0445-09.2009

67. Bonato C, Miniussi C, Rossini PM (2006) Transcranial magnetic stimulation and cortical evoked potentials: a TMS/EEG coregistration study. Clin Neurophysiol 117:1699-1707

68. Lioumis P, Kicić D, Savolainen P, Mäkelä JP, Kähkönen S (2009) Reproducibility of TMS-evoked EEG responses. Hum Brain Mapp 30(4):1387-1396. https://doi.org/10.1002/hbm.20608

69. Nikouline V, Ruohonen J, Ilmoniemi RJ (1999) The role of the coil click in TMS assessed with imultaneous. EEG Clin Neurophysiol 110:1325-1328

70. Tremblay S, Rogasch NC, Premoli I, Blumberger DM, Casarotto S, Chen R (2019) Clinical utility and prospective of TMS-EEG. Clin Neurophysiol 12:4534-4577

71. Massimini M, Ferrarelli F, Huber R, Esser SK, Singh H, Tononi G (2005) Breakdown of cortical effective connectivity during sleep. Science 309(5744):2228-2232

72. Premoli I, Castellanos N, Rivolta D, Belardinelli P, Bajo R, Zipser C, Espenhahn S, Heidegger T, Müller-Dahlhaus F, Ziemann U (2014) TMS-EEG signatures of GABAergic neurotransmission in the human cortex. J Neurosci 34:5603-5612

73. Zanon M, Battaglini PP, Jarmolowska J, Pizzolato G, Busan P (2013) Long-range neural activity evoked by premotor cortex stimulation: a TMS/EEG co-registration study. Front Hum Neurosci 7:803. https://doi.org/10.3389/fnhum.2013.00803

74. Fecchio M, Pigorini A, Comanducci A, Sarasso S, Casarotto S, Premoli I, Derchi CC, Mazza A, Russo S, Resta F, Ferrarelli F, Mariotti M, Ziemann U, Massimini M, Rosanova (2017) The 
spectral features of EEG responses to transcranial magnetic stimulation of the primary motor cortex depend on the amplitude of the motor evoked potentials. PLoS One 12(9):e0184910. https://doi. org/10.1371/journal.pone.0184910

75. Salo VC, Ferrari PF, Fox NA (2019) The role of the motor system in action understanding and communication: evidence from human infants and non-human primates. Dev Psychobiol 61(3):390 401

76. Pisoni A, Mattavelli G, Papagno P, Rosanova M, Casali AG, Romero Lauro LJ (2018) Cognitive enhancement induced by anodal tDCS drives circuit-specific cortical plasticity. Cereb Cortex 28:1132-1140

77. Koch G, Bonnì S, Pellicciari MC, Casula EP, Mancini M, Esposito R, Ponzo V, Picazio S, Di Lorenzo F, Serra L, Motta C, Maiella M, Marra C, Cercignani M, Martorana A, Caltagirone C, Bozzali M (2018) Transcranial magnetic stimulation of the precuneus enhances memory and neural activity in prodromal Alzheimer's disease. Neuroimage 1(169):302-311. https://doi. org/10.1016/j.neuroimage.2017.12.048

78. Koch G, Bonnì S, Casula EP, Iosa M, Paolucci S, Pellicciari MC et al (2019) Effect of cerebellar stimulation on gait and balance recovery in patients with hemiparetic stroke: a randomized clinical trial. JAMA Neurol 76:170-178

79. Zazio A, Bortoletto M, Ruzzoli M, Miniussi C, Veniero D (2019) Perceptual and physiological consequences of dark adaptation: a TMS-EEG study. Brain Topogr 32:773-782. https://doi.org/10. 1007/s10548-019-00715-x

80. Bergmann TO, Karabanov A, Hartwigsen G, Thielscher A, Siebner HR (2016) Combining non-invasive transcranial brain stimulation with neuroimaging and electrophysiology: current approaches and future perspectives. Neuroimage 140:4-19

81. Bortoletto M, Veniero D, Thut G, Miniussi C (2015) The contribution of TMS-EEG coregistration in the exploration of the human cortical connectome. Neurosci Biobehav Rev 49:114-124. https://doi.org/10.1016/j.neubiorev.2014.12.014

82. Farzan F, Barr MS, Levinson AJ, Chen R, Wong W, Fitzgerald PB, Daskalakis ZJ (2010) Reliability of long interval cortical inhibition in healthy human subjects: a TMSEEG study. J Neurophysiol 104:1339-1346

83. Koch G, Ponzo V, Di Lorenzo F, Caltagirone C, Veniero D (2013) Hebbian and anti-hebbian spike-timing-dependent plasticity of human cortico-cortical connections. J Neurosci 33:9725-9733

84. Casula EP, Pellicciari MC, Picazio S, Caltagirone C, Koch G (2016) Spike-timing-dependent plasticity in the human dorsolateral prefrontal cortex. Neuroimage 143:204-213

85. Ragazzoni A, Cincotta M, Giovannelli F, Cruse D, Young GB, Miniussi C, Rossi S (2017) Clinical neurophysiology of prolonged disorders of consciousness: from diagnostic stimulation to therapeutic neuromodulation. Clin Neurophysiol 128(9):1629-1646

86. Sun Y, Farzan F, Mulsant BH, Rajji TK, Fitzgerald PB, Barr MS, Downar J, Wong W, Blumberger DM, Daskalakis ZJ (2016) Indicators for remission of suicidal ideation following magnetic seizure therapy in patients with treatment-resistant depression. JAMA Psychiatry 73:337-345

87. Casarotto S, Canali P, Rosanova M, Pigorini A, Fecchio M, Mariotti M, Lucca A, Colombo C, Benedetti F, Massimini M (2013) Assessing the effects of electroconvulsive therapy on cortical excitability by means of transcranial magnetic stimulation and electroencephalography. Brain Topogr 26(2):326-337. https://doi.org/10.1007/s10548-012-0256-8

88. Pellicciari MC, Ponzo V, Caltagirone C, Koch G (2017) Restored asymmetry of prefrontal cortical oscillatory activity after bilateral theta burst stimulation treatment in a patient with major depressive disorder: a TMS-EEG study. Brain Stimul 10:147-149

89. Pellicciari MC, Bonnì S, Ponzo V, Cinnera AM, Mancini M, Casula EP, Sallustio F, Paolucci S, Caltagirone C, Koch G
(2018) Dynamic reorganization of TMS-evoked activity in subcortical stroke patients. Neuroimage 175:365-378

90. Bagattini C, Mutanen TP, Fracassi C, Manenti R, Cotelli M, Ilmoniemi RJ, Miniussi C, Bortoletto M (2019) Predicting Alzheimer's disease severity by means of TMS-EEG coregistration. Neurobiol Aging 80:38-45

91. Mutanen TP, Kukkonen M, Nieminen JO, Stenroos M, Sarvas J, Ilmoniemi RJ (2016) Recovering TMS-evoked EEG responses masked by muscle artifacts. Neuroimage 139:157-166

92. Mutanen TP, Metsomaa J, Liljander S, Ilmoniemi RJ (2018) Automatic and robust noise suppression in EEG and MEG: the SOUND algorithm. Neuroimage 166:135-151

93. Conde V, Tomasevic L, Akopian I, Stanek K, Saturnino GB, Thielscher A, Bergmann TO, Siebner HR (2019) The nontranscranial TMS evoked potential is an inherent source of ambiguity in TMS-EEG studies. Neuroimage 185:300-312

94. Belardinelli P, Biabani M, Blumberger DM, Bortoletto M, Casarotto S, David O, Desideri D, Etkin A, Ferrarelli F, Fitzgerald PB, Fornito A, Gordon PC, Gosseries O, Harquel S, Julkunen P, Keller CJ, Kimiskidis VK, Lioumis P (2019) Reproducibility in TMS-EEG studies: a call for data sharing, standard procedures and effective experimental control. Brain Stimul 12:787-790

95. Siebner HR, Conde V, Tomasevic L, Thielscher A, Bergmann TO (2019) Distilling the essence of TMS-evoked EEG potentials (TEPs): a call for securing mechanistic specificity and experimental rigor. Brain Stimul 12:1051-1054. https://doi.org/10.1016/j. brs.2019.03.076

96. Zrenner C, Belardinelli P, Müller-Dahlhaus F, Ziemann U (2016) Closed-loop neuroscience and non-invasive brain stimulation: a tale of two loops. Front Cell Neurosci 10:92. https://doi.org/10. 3389/fncel.2016.00092

97. Cohen D, Cuffin BN, Yunokuchi K, Maniewski R, Purcell C, Cosgrove GR, Ives J, Kennedy JG, Schomer DL (1990) MEG versus EEG localization test using implanted sources in the human brain. Ann Neurol 28(6):811-817

98. Baillet S (2017) Magnetoencephalography for brain electrophysiology and imaging. Nat Neurosci 20(3):327-339. https://doi.org/ 10.1038/nn.4504

99. Hari R, Puce A, Baillet S, Barnes G, Burgess R, Forss N, Gross J (2018) IFCN-endorsed practical guidelines for clinical magnetoencephalography (MEG). Clin Neurophysiol 129(8):1720-1747. https://doi.org/10.1016/j.clinph.2018.03.042

100. Parkkonen L (2010) Instrumentation and data preprocessing. In: Hansen C, Peter M, Kringelbach L, Salmelin R (eds) MEG: an introduction to methods. Oxford university press, New York, pp 24-64

101. Hulten A, Schoffelen J, Udd J, Lam NHL, Hagoort P (2019) NeuroImage How the brain makes sense beyond the processing of single words - an MEG study. Annika Hult 186:586-594. https://doi.org/10.1016/j.neuroimage.2018.11.035

102. van Es MWJ, Van Schoffelen JS (2019) Stimulus-induced gamma power predicts the amplitude of the subsequent visual evoked response. NeuroImage 186:703-712. https://doi.org/10.1016/j. neuroimage.2018.11.029

103. Näätänen R, Pakarinen S, Rinne T, Takegata R (2004) The mismatch negativity (MMN): towards the optimal paradigm. Clin Neurophysiol 115(1):140-144. https://doi.org/10.1016/j.clinph. 2003.04.001

104. Recasens M, Uhlhaas PJ (2017) Test-retest reliability of the magnetic mismatch negativity response to sound duration and omission deviants. NeuroImage 157:184-195. https://doi.org/10.1016/ j.neuroimage.2017.05.064

105. Thönnessen H, Zvyagintsev M, Harke KC, Boers F, Dammers J, Norra C, Mathiak K (2008) Optimized mismatch negativity paradigm reflects deficits in schizophrenia patients. A combined EEG 
and MEG study. Biol Psychol 77(2):205-216. https://doi.org/10. 1016/j.biopsycho.2007.10.009

106. Tiitinen H, Alho K, Huotilainen M, Ilmoniemi RJ, Simola J, Näätänen R (1993) Tonotopic auditory cortex and the magnetoencephalographic (MEG) equivalent of the mismatch negativity. Psychophysiology 30(5):537-540

107. Hsiao FJ, Wu ZA, Ho LT, Lin YY (2009) Theta oscillation during auditory change detection: an MEG study. Biol Psychol 81(1):58 66. https://doi.org/10.1016/j.biopsycho.2009.01.007

108. Rossini PM, Tecchio F, Pizzella V, Lupoi D, Cassetta E, Pasqualetti P, Orlacchio A (1998) On the reorganization of sensory hand areas after mono-hemispheric lesion: a functional (MEG)/anatomical (MRI) integrarive study. Brain Res 782(12):153-166. https://doi.org/10.1016/S0006-8993(97)01274-2

109. Polich J, Kok A (1995) Cognitive and biological determinants of P300: an integrative review. Biol Psychol 41(2):103-146

110. Polich J (2007) Updating P300: an integrative theory of P3a and P3b. Clin Neurophysiol 118(10):2128-2148. https://doi.org/10. 1016/j.clinph.2007.04.019

111. Mecklinger A, Maess B, Opitz B, Pfeifer E, Cheyne D, Weinberg $\mathrm{H}$ (1998) A MEG analysis of the P300 in visual discrimination tasks. Electroencephalogr Clin Neurophysiol Evoked Potent 108(1):45-56. https://doi.org/10.1016/S0168-5597(97)00092-0

112. Linden DEJ (2005) The P300: where in the brain is it produced and what does it tell us? Neuroscientist 11(6):563-576. https://doi. org/10.1177/1073858405280524

113. Bagić AI, Knowlton RC, Rose DF, Ebersole JS (2011) American Clinical Magnetoencephalography Society Clinical Practice Guideline 1: recording and analysis of spontaneous cerebral activity*. J Clin Neurophysiol 28(4):348-354. https://doi.org/10.1097/ WNP.0b013e3182272fed

114. Burgess RC, Funke ME, Bowyer SM, Lewine JD, Kirsch HE, Bagić AI (2011) American Clinical Magnetoencephalography Society Clinical Practice Guideline 2: presurgical functional brain mapping using magnetic evoked fields*. J Clin Neurophysiol 28(4):355-361. https://doi.org/10.1097/WNP.0b013e3182272ffe

115. Hari R, Baillet S, Barnes G, Burgess R, Forss N, Gross J, Taulu S (2018) IFCN-endorsed practical guidelines for clinical magnetoencephalography (MEG). Clin Neurophysiol 129:1720-1747. https://doi.org/10.1016/j.clinph.2018.03.042

116. Pellegrino G, Hedrich T, Chowdhury R, Hall JA, Lina J, Dubeau F, Grova C (2016) Source localization of the seizure onset zone from ictal EEG / MEG Data. 2546:2528-2546. https://doi.org/10. 1002/hbm.23191

117. Pellegrino G, Hedrich T, Chowdhury RA, Hall JA, Dubeau F, Lina JM, Grova C (2018) Clinical yield of magnetoencephalography distributed source imaging in epilepsy: a comparison with equivalent current dipole method. Hum Brain Mapp 39(1):218231. https://doi.org/10.1002/hbm.23837
118. Hedrich T, Pellegrino G, Kobayashi E, Lina JM, Grova C (2017) Comparison of the spatial resolution of source imaging techniques in high-density EEG and MEG. NeuroImage 157:531-544. https://doi.org/10.1016/j.neuroimage.2017.06.022

119. Ottenhausen M, Krieg SM, Meyer B, Ringel F (2015) Functional preoperative and intraoperative mapping and monitoring: increasing safety and efficacy in glioma surgery. 38:1-13. https://doi.org/ 10.3171/2014.10.FOCUS14611.Disclosure

120. Frye RE, Rezaie R, Papanicolaou AC (2009) Functional neuroimaging of language using magnetoencephalography. Phys Life Rev 6(1):1-10. https://doi.org/10.1016/j.plrev.2008.08.001

121. Papanicolaou C, Simos PG, Breier JI, Zouridakis G, Willmore LJ, Wheless JW, Gormley WB (1999) Magnetoencephalographic mapping of the language-specific cortex. J Neurosurg 90(1):8593. https://doi.org/10.3171/jns.1999.90.1.0085

122. Pang EW, Wang F, Malone M, Kadis DS, Donner EJ (2011) Localization of Broca's area using verb generation tasks in the MEG: validation against fMRI. Neurosci Lett 490(3):215-219. https://doi.org/10.1016/j.neulet.2010.12.055

123. Salmelin R (2007) Clinical neurophysiology of language: the MEG approach. Clin Neurophysiol 118(2):237-254. https://doi. org/10.1016/j.clinph.2006.07.316

124. Betti V, Zappasodi F, Rossini PM, Aglioti SM, Tecchio F (2009) Synchronous with your feelings: sensorimotor \{gamma\} band and empathy for pain. J Neurosci 29(40):12384-12392. https://doi. org/10.1523/JNEUROSCI.2759-09.2009

125. Betti V, Della Penna S, de Pasquale F, Mantini D, Marzetti L, Romani GL, Corbetta M (2013) Natural scenes viewing alters the dynamics of functional connectivity in the human brain. Neuron 79(4):782-797. https://doi.org/10.1016/j.neuron.2013. 06.022

126. Pellegrino G, Maran M, Turco C, Weis L, Pino G, Di Piccione F, Arcara G (2018) Bilateral transcranial direct current stimulation reshapes resting-state brain networks: a magnetoencephalography assessment. Neural Plast 2018:2782804. https://doi.org/10.1155/ $2018 / 2782804$

127. Sorrentino P, Rucco R, Jacini F, Trojsi F, Lardone A, Baselice F, Sorrentino G (2018) Brain functional networks become more connected as amyotrophic lateral sclerosis progresses: a source level magnetoencephalographic study. NeuroImage 20:564-571. https://doi.org/10.1016/j.nicl.2018.08.001

128. Paggiaro A, Birbaumer N, Cavinato M, Turco C, Formaggio E, Del Felice A, Piccione F (2016) Magnetoencephalography in stroke recovery and rehabilitation. Front Neurol 7:35

Publisher's note Springer Nature remains neutral with regard to jurisdictional claims in published maps and institutional affiliations. 